\title{
LA CLASSIFICAZIONE ARISTOTELICA DELLE SCIENZE IN PIETRO D’ABANO
}

\section{Enrico Berti ${ }^{1}$}

\begin{abstract}
RIASSUNTO: La classificazione delle scienze di Pietro d'Abano costituisce un'interessante riformulazione della classificazione analoga, proposta da Aristotele in Metaph. VI, e della teoria degli abiti dianoetici, proposta da Aristotele in Eth. Nic. VI. Come risulta dal Conciliator per quanto concerne la medicina e dal Lucidator per quanto concerne l'astronomia, Pietro segue la classificazione aristotelica e le interpretazioni che di essa erano state date nel medioevo (dottrina dei tre gradi di astrazione e distinzione tra methodus compositiva e methodus resolutiva), aggiungendovi come contributo originale l'introduzione di una parte pratica sia nella medicina che nell'astronomia (astronomia iudicialis), dove quest'ultima deriva da Tolomeo e dagli Arabi.
\end{abstract}

PAROLE CHIAVE: Aristotele, scienza, arte, medicina, astronomia, astrologia.

\section{SCIENZE E ABITI DiANOETICI IN ARISTOTELE}

La classificazione delle scienze esposta da Aristotele nel VI libro della Metafisica è uno degli aspetti del suo pensiero che hanno avuto maggiore fortuna e che hanno più a lungo influito sull'organizzazione della cultura nel medioevo e nell'età moderna. Essa è presente, naturalmente, anche nelle opere di Pietro d'Abano (1250 circa-1315 circa), il fondatore - secondo Renan della cosiddetta "Scuola di Padova", dove viene interpretata con alcuni tratti di originalità, che meritano di essere sottolineati. Ricordiamo anzitutto nelle sue linee generali la suddetta classificazione. Per Aristotele la "ragione" (dianoia), intesa come sinonimo di "scienza", può essere "teoretica", "pratica" o "poietica". Una scienza è teoretica quando verte su un oggetto che non ha come principio l'uomo, è pratica quando ha per oggetto un'azione (praxis), attività umana che non termina in altro, ed è poietica quando ha per oggetto una produzione (poiêsis), cioè un'attività umana che termina in un prodotto. Sono pertanto scienze teoretiche la "fisica", che ha per oggetto gli enti naturali, realtà mobili e quindi non separate dalla materia, le "matematiche", che hanno per oggetto realtà non separate dalla materia, ma considerate come se lo

\footnotetext{
${ }^{1}$ Dipartimento di Filosofia, Sociologia, Pedagogia e Psicologia Applicata. Università degli Studi di Padova.
}

${ }^{2}$ RENAN, 1861. 
fossero e quindi come immobili, e la "filosofia prima", che, ricercando le cause prime dell'essere in quanto essere, e quindi anche degli astri, attinge a realtà immobili e separate dalla materia, cioè divine, e quindi può essere detta anche "scienza teologica" ${ }^{3}$ Quest'ultima scienza, esposta nell'opera edita col titolo di Metafisica, sarà detta anche "metafisica". I commentatori neoplatonici di Aristotele collocheranno tra le scienze pratiche l'etica, concernente il bene del singolo, l'economica, concernente il bene della famiglia (oikos), e la politica, concernente il bene della città (polis); gli stessi collocheranno tra le scienze poietiche l'arte retorica, l'arte poetica e, almeno alcuni, l'arte dialettica, cioè la logica, e quindi le arti (technai) ${ }^{4}$.

La giustificazione di questa classificazione è contenuta nel VI libro dell'Etica Nicomachea, dove Aristotele tratta dei cosiddetti "abiti dianoetici", cioè le disposizioni abituali della ragione, allo scopo di individuare tra essi le "virtù dianoetiche", cioè le eccellenze della ragione. Egli anzitutto divide la parte razionale dell'anima, cioè appunto la ragione, in due parti, quella che verte sugli "enti i cui princìpi non possono stare diversamente" e quella che verte sugli enti che invece possono stare diversamente: i primi sono gli enti naturali, che di per se stessi possono stare diversamente da come stanno, cioè sono mobili, o contingenti, ma hanno princìpi, cioè cause prime, che sono realtà immobili, cioè necessarie; i secondi sono gli enti che dipendono dall'uomo, cioè le azioni e le produzioni, che abbiamo già menzionato. La parte della ragione che considera gli enti naturali è detta "scientifica" (epistêmonikon), mentre quella che considera le attività umane è detta "calcolatrice" (logistikon), perché le attività umane sono frutto di una deliberazione, la quale per Aristotele suppone un calcolo. Esse inoltre sono anche dette rispettivamente "ragione teoretica" e "ragione pratica"5.

Gli abiti dianoetici, per mezzo dei quali - dice Aristotele - l'anima è nel vero quando afferma e quando nega, sono cinque: arte (technê), scienza (epistêmê), saggezza (phronêsis), sapienza (sophia), intelletto (nous). La scienza è un abito della ragione scientifica, o teoretica, che verte sulle realtà i cui princìpi non possono stare diversamente, ed è precisamente l'abito che permette di fare dimostrazioni, cioè di dimostrare, a partire da princìpi immutabili, le proprietà necessarie dei suoi oggetti. L'arte invece è un abito della ragione calcolatrice o pratica, che verte sulle produzioni di oggetti, e quindi è l'abito che permette alla ragione di produrre oggetti per mezzo di calcoli veri, come per esempio fa

\footnotetext{
${ }^{3}$ ARISTOTELIS, Metaphysica, VI 1, 1025 b 21-1026 a 25.

${ }^{4}$ ARISTOTELIS, Opera, III, p. 61-90.

${ }^{5}$ ARISTOTELIS, Ethica Nicomachea, VI 2, 1139 a 1-b 5.
} 
l'architettura. La saggezza, poi, è anch'essa un abito della ragione pratica, che però verte sulle azioni, e ha come fine l'agire bene, cioè il bene del singolo, o della famiglia, o della città: un esempio di uomo saggio è quindi il buon politico, Pericle. Poiché l'azione, in quanto fine a se stessa, è superiore alla produzione, che ha il suo fine in altro, la saggezza è superiore all'arte ed è quindi la virtù, cioè l'eccellenza, della ragione pratica. La scienza, dal canto suo, in quanto abito dimostrativo, presuppone il possesso di princìi da cui dimostrare, e l'abito dei princìpi, cioè lo stato in cui si trova abitualmente colui che possiede i princìpi, è l'intelletto. L'insieme di intelletto e scienza, cioè di conoscenza dei princìpi e di capacità di dimostrare a partire da questi, è per Aristotele la sapienza, che pertanto viene ad essere la virtù, ovvero l'eccellenza, della ragione teoretica. Poiché l'oggetto della sapienza comprende anche realtà divine, quali erano per Aristotele gli astri, che sono superiori all'uomo, la sapienza è anche superiore alla saggezza, cioè è il bene supremo per l'uomo, nel cui esercizio consiste la felicità .

Non è difficile conciliare la classificazione degli abiti dianoetici dell' Etica con la classificazione delle scienze della Metafisica. Le scienze teoretiche, menzionate in quest'ultima, rientrano infatti nell'abito della scienza, che verte sulle realtà i cui princìpi sono necessari, e quindi comprende la fisica, che verte su realtà mobili, ma le cui cause prime (materia prima, forme sostanziali e primi motori) sono immobili, e le matematiche, sia le matematiche pure (aritmetica e geometria) sia quelle applicate (astronomia, meccanica, ottica e armonica), i cui principi sono definizioni, assiomi e postulati. La filosofia prima, o scienza teologica, o metafisica, coincide poi con poi l'abito della sapienza. Le scienze pratiche hanno uno statuto meno chiaro: in quanto scienze, infatti, esse dovrebbero rientrare nell'abito della scienza, che è un abito della ragione scientifica, ma in quanto vertono sulle azioni, che possono stare diversamente, esse dovrebbero essere un abito della ragione calcolatrice, o pratica. La difficoltà si risolve, a mio avviso, se si distinguono le vere e proprie scienze pratiche, come la scienza politica - la quale secondo Aristotele abilita a fare delle dimostrazioni, che però valgono non sempre (come quelle delle matematiche), ma solo "per lo più" (come quelle della fisica) ${ }^{7}$-, dalla saggezza, la quale abilita a fare non dimostrazioni, ma deliberazioni, cioè calcoli, come il cosiddetto sillogismo pratico, e non è abito del filosofo, sia pure morale (come Socrate), ma del politico (come Pericle). Quanto alle scienze poietiche, si può

${ }^{6}$ Ivi, 1139 b $14-1141$ b 8.

${ }^{7}$ Come Aristotele spiega in Ethica Nicomachea, I 1, 1094 b 20-25. 
ammettere che esse rientrino nell'arte, come hanno sostenuto i commentatori, anche perché Aristotele stesso, nella Metafisica, dichiara che degli oggetti di tali scienze è principio l'arte ${ }^{8}$.

In ogni caso, scienza e arte per Aristotele sono abiti diversi, appartenenti a due diversi tipi di ragione, l'una teoretica e l'altra pratica, anche se sono entrambi abiti razionali, cioè capacità di conoscere razionalmente, e come tali hanno anche delle affinità. Queste sono illustrate da Aristotele nel celebre inizio della Metafisica, dove egli spiega il passaggio dal senso (aisthêsis) alla memoria (mnêmê), da questa all'esperienza (empeiria) e dall'esperienza alla scienza e all'arte, qui menzionate insieme e collocate al medesimo livello di conoscenza. Il passaggio dall'esperienza alla scienza e all'arte - spiega Aristotele - avviene quando da molti contenuti di esperienza si genera un unico giudizio universale riguardante i casi simili, e tale passaggio viene da lui illustrato nel modo seguente: sapere che una certa cosa (per esempio un farmaco, o una dieta, o un clima) ha giovato a Callia ammalato di una certa malattia, e a Socrate e a molti altri individui ammalati della stessa malattia, è esperienza; sapere invece che tale cosa ha giovato a tutti gli ammalati di tale malattia, definiti secondo un'unica specie, o forma (eidos), per esempio a tutti i flemmatici o a tutti i biliosi malati di febbre ardente, è arte. L'esperienza, infatti, è conoscenza del particolare, e del "che", cioè del dato di fatto, mentre l'arte e la scienza sono conoscenza dell'universale, e del "perché", cioè della causa, della ragione, della spiegazione9. Qui Aristotele non solo colloca sullo stesso piano la scienza e l'arte, ma per spiegare il passaggio dall'esperienza ad esse si serve di un esempio tratto dalla medicina, cioè da un'arte, l'arte di produrre la salute.

\section{Lo STATUTO EPISTEMOlOGiCo DELLA MEDICINA NEL CONCILIATOR}

La classificazione aristotelica delle scienze, anzi, prima ancora, la classificazione aristotelica degli abiti dianoetici, esposta nell'Etica Nicomachea, viene ripresa da Pietro d'Abano nel Conciliator, cioè nell'opera che, come risulta dal titolo, si propone di conciliare le differenze tra i filosofi, ossia soprattutto Aristotele e i suoi commentatori, e i medici, cioè Ippocrate, Galeno e i medici arabi. Nella Differentia III di quest'opera, che verte sul tema utrum medicina sit scientia, necne, Pietro anzitutto riporta gli argomenti contro la scientificità della medicina, cioè: 1) la tesi, sostenuta da Aristotele negli Analitici posteriori,

\footnotetext{
${ }^{8}$ ARISTOTELIS, Metaphysica, VI 1,1025 b 21-23.

${ }^{9}$ ARISTOTELIS, Metaphysica, I 1, 981 a 1-30.
} 
secondo cui la scienza deve avere oggetti incorruttibili ed eterni, mentre la medicina ha per oggetto il corpo umano, mutevole e corruttibile; 2) la tesi secondo cui la scienza deve avere oggetti astratti, mentre la medicina ha oggetti concreti (i malati e i sani); 3) la tesi secondo cui la scienza deve essere degli universali, mentre la medicina è dei particolari, perché cura non l'uomo in generale, ma Socrate, Callia e altri individui; 4) infine la tesi secondo cui una scienza subalterna, come sembra essere la medicina, dovrebbe essere posta sotto una scienza superiore, mentre la medicina sembra essere posta piuttosto sotto le arti meccaniche, perché il suo fine è la salute, la quale viene prodotta, come accade nelle arti meccaniche ${ }^{10}$.

Contro tali argomenti Pietro cita il fatto che Aristotele, Galeno e altri medici e filosofi hanno considerato la medicina come una scienza e pertanto si accinge a difendere questa tesi, rispondendo agli argomenti sopra riportati. Per fare questo, egli si richiama esplicitamente alla distinzione tra i cinque abiti per mezzo dei quali l'anima dice il vero affermando o negando, esposta da Aristotele nel VI libro dell'Etica Nicomachea, cioè scientia, ars, prudentia, sapientia, intellectus. A proposito della scientia, Pietro distingue: 1) una scientia propriissime dicta, cioè quella che, partendo da cause prossime e immediate, dimostra le sue conclusioni, come dice Aristotele nel I libro degli Analitici posteriori. Secondo Pietro, questa scienza coincide con quella che Galeno chiama doctrina compositiva. 2) Una scienza che può essere detta propriissime quoad nos, cioè quella che procede dalle cose più note a noi verso quelle più note per natura, ovvero dagli effetti alle cause, di cui Aristotele parla all'inizio della Fisica, che secondo Pietro può essere detta doctrina resolutiva. 3) Una scienza intesa improprie \& communiter, cioè la comprensione della verità per mezzo della ragione o dell'esperienza. Pietro poi conclude che la scienza in assoluto è la comprensione, da parte dell'anima, delle cose preparate ad essere sapute, la quale è utile all'acquisizione della felicità eterna, come dice Aristotele nel I libro della Metafisica ${ }^{11}$.

L'assimilazione delle prime due accezioni aristoteliche della scienza la cui distinzione ha un fondamento nella distinzione, fatta negli Analitici posteriori, tra la dimostrazione del "perché" e la dimostrazione del "che"12 - , alla doctrina compositiva e alla doctrina resolutiva distinte da Galeno nell'Ars medica (detta anche Ars parva, o con un calco dal greco Microtegni o

\footnotetext{
${ }^{10}$ PETRI DE ABANO, Conciliator, f. 5 v.

${ }^{11}$ Conc., f. 6 r.

${ }^{12}$ ARISTOTELIS, Analytica et posteriora, I 13, 78 a 21-b 4.
} 
semplicemente Tegni), era consueta nel medioevo ${ }^{13}$ e avrebbe continuato ad esserlo nel rinascimento (per esempio in Zabarella), anche se essa è del tutto ingiustificata dal punto di vista di Aristotele, per il quale l'analisi (in latino resolutio) è un procedimento matematico che non ha nulla a che fare con la dimostrazione del "che" 14 . Ma non è a questo proposito, cioè a proposito della scienza, che Pietro manifesta la sua originalità rispetto alla classificazione aristotelica, bensì a proposito del secondo abito dianoetico menzionato da Aristotele, cioè l'arte.

Anzitutto Pietro richiama la definizione dell'arte data da Aristotele nel VI libro dell'Etica Nicomachea, cioè "abito produttivo con retta ragione" (habitus cum recta ratio factivus), ovvero "retta ragione delle cose producibili" (recta ratio factibilium), osservando che essa si genera quando "da molte concezioni sperimentali si produce un unico giudizio universale sui casi simili”, che è esattamente la descrizione del passaggio dall'esperienza all'arte e alla scienza contenuta nel I libro della Metafisica. Poi egli introduce subito il problema che più gli sta a cuore, cioè lo statuto della medicina, osservando che questa talvolta è detta scienza e talvolta invece arte, ma anche che tra arte e scienza sembra esservi una distanza non piccola (appareat distare non parum), poiché la scienza sembra essere qualcosa che è essere in facto, mentre l'arte è piuttosto qualcosa in fieri, come dice Aristotele - secondo Pietro - alla fine degli Analitici posteriori ${ }^{15}$. Per sottolineare tale distanza Pietro richiama il fondamento della distinzione aristotelica tra scienza e arte, cioè il fatto che l'arte si occupa di cose il cui principio è nell'uomo che fa (in faciente), cioè in altro, mentre la scienza si occupa di cose il cui principio è nella natura, cioè nelle stesse cose naturali, ed a questo proposito egli ricorda la distinzione fra le scienze teoretiche esposta nel VI libro della Metafisica, dicendo che i modi essentiales della filosofia sono tre, cioè naturalis, mathematicus, \& divinus ${ }^{16}$.

A questo punto però Pietro intraprende un ragionamento del tutto originale, cioè: "Si deve tuttavia tener presente che l'arte può essere considerata in due modi, o in quanto è abito acquisito nell'anima, e così è essere di fatto, come anche la scienza, o in quanto si riferisce a cose fattibili, a proposito delle quali essa è nel farle, e così è detta essere intorno alla generazione, e divenire; la

\footnotetext{
${ }^{13} \mathrm{Si}$ veda un riassunto dello status quaestionis in SELLER, p. 21-32.

${ }^{14}$ BERTI, 2009.

${ }^{15}$ ARISTOTELIS, Analytica et posteriora, II 19, 100 a 8-9, dove però si dice che l'arte è "intorno alla generazione" (peri genesin), mentre la scienza è "intorno all'ente" (peri to on).

${ }^{16}$ Conc., f. 6 r.
} 
scienza invece in entrambi i modi è detta nell'essere, e nel fatto già perfetto"17. Il discorso è ambiguo, perché sembra passare dall'affermazione che l'arte verte su cose in divenire, ripresa da Aristotele, all'affermazione che l'arte è essa stessa in divenire, mentre la scienza non solo verte sull'essere, ma si trova essa stessa in un essere del tutto compiuto, cioè non diveniente. E Pietro prosegue: "Poiché dunque, come apparirà, la medicina ha due parti, cioè una teorica e una pratica, quanto alla teorica può essere detta propriamente scienza, mentre quanto alla pratica è piuttosto arte. E perché Ippocrate, secondo Galeno, fu inventore della medicina, infatti fu anteriore a tutti quelli che seppe, ed inventore della scienza, quasi in movimento, e stette maggiormente nel fare, ora con l'applicare sé alle cose mediante l'esperimento, ora infine mediante la ragione, non avendo fino a questo momento un abito perfetto, e quietato nell'anima" ${ }^{18}$. Da queste parole si ricava l'impressione che la parte pratica della medicina, in base alla quale essa è un'arte, preceda quella teorica, in base alla quale essa è una scienza, e consista nel fare per mezzo di esperimenti, sino a giungere ad essere, per mezzo della ragione, un abito perfetto, non più in divenire, ma in quiete ${ }^{19}$.

Nel seguito della risposta agli argomenti contro la scientificità della medicina Pietro illustra anche gli altri tre abiti dianoetici descritti da Aristotele nell'Etica Nicomachea, cioè la prudentia, la sapientia e l'intellectus, riportando fedelmente il pensiero di Aristotele. Egli poi mostra che anche per Aristotele è possibile avere scienza di oggetti corruttibili, come sono i corpi sensibili, perché l'intelletto può astrarre da essi la forma intelligibile, che è incorruttibile ed universale, e della quale dunque ci può essere scienza. In tal modo egli risponde alle obiezioni secondo cui la scienza deve avere per oggetto enti incorruttibili, astratti ed universali, servendosi di dottrine aristoteliche, interpretate secondo la tradizione della filosofia scolastica. La medicina quindi, conclude Pietro, corrisponde perfettamente ai requisiti che, secondo

\footnotetext{
17 "Est tamen advertendum, quod ars potest dupliciter considerari: aut prout est habitus acquisitus in anima: $\&$ sic est in facto esse sicut $\&$ scientia: vel prout refertur ad res factibiles, de quibus est ea faciendo: $\&$ ita esse dicitur circa generationem, $\&$ fieri: scientia vero utroque modo dicitur in esse, $\&$ in facto iam perfecto".

18 "Cum igitur, ut apparebit, medicina duas habeat partes: theoricam scilicet, \& practicam: quantum ad theoricam potest scientia proprius dici: quo vero ad practicam ars magis, \& quia Hippocrates, secundum Galenum, fuit medicinae inventor: omnibus enim quos scivit prior, \& inventor scientiae, quasi in motu, \& factione amplius extitit applicando nunc se rebus esperimento: demum vero ratione, cum adhuc habitum non haberet perfectum, \& quietatum in anima”.

${ }^{19}$ Ulteriori chiarimenti sulla medicina come sintesi di arte e scienza si possono trovare in FEDERICI VESCOVINI, 1999.
} 
Aristotele, una scienza deve possedere, perché ha un oggetto unitario, il corpo umano, cioè il più nobile fra tutti i corpi, il quale è un ente per sé, ma ha anche delle passiones, che possono essere dimostrate, e delle causae sufficientes. Le dimostrazioni della medicina non sono sempre dimostrazioni certe, ma spesso si servono di congetture che si avvicinano alla realtà ${ }^{20}$.

È forse vero dunque, come è stato sostenuto, che Pietro d'Abano tratteggia un concetto di scienza diverso da quello prevalentemente accettato dall'aristotelismo medievale, tomistico o averroistico, secondo cui c'è scienza solo delle sostanze eterne, immutabili e perfette ${ }^{21}$. Ma il suo concetto di scienza è perfettamente aristotelico, perché Aristotele ammetteva anche scienze di realtà corruttibili e mobili, quali gli enti naturali, oggetto della fisica, e di realtà contingenti, quali le azioni e le produzioni umane, oggetto delle scienze pratiche e poietiche, assegnando ad entrambi questi tipi di scienza dimostrazioni valide non sempre, ma "per lo più", cioè nella maggior parte dei casi $^{22}$. Non è vero, invece, che egli avesse abbandonato uno dei capisaldi della metafisica aristotelica, cioè - come è stato ugualmente sostenuto - la concatenazione causale necessaria, per cui dall'effetto si può risalire alla causa e viceversa ${ }^{23}$, perché questo non era affatto un caposaldo della metafisica aristotelica, la quale non ammetteva in natura alcuna concatenazione causale necessaria, né la possibilità di risalire sempre dall'effetto alla causa e viceversa ${ }^{24}$.

All'obiezione secondo cui la medicina non sembra essere subalterna a nessuna scienza, ma piuttosto alle arti meccaniche, Pietro risponde che, come l'astronomia è subalterna alle scienze matematiche, in particolare all'aritmetica e alla geometria (su questo ritorneremo), così la medicina è subalterna ad una scienza quale la fisica, perché il corpo umano, suo oggetto, è un ente naturale, per il quale valgono dunque i princìpi della scienza naturale, che sono princìi veri: "medicina - egli afferma - est ars operatrix exiens ex principiis veris, scilicet naturalibus" 25 . Anche in questo egli è perfettamente in linea con Aristotele, il quale più volte afferma che spetta al fisico conoscere i princìi della salute e della malattia, perché la salute e la malattia appartengono ad esseri viventi, quindi naturali, perciò la maggior parte dei fisici finiscono

\footnotetext{
${ }^{20}$ Conc., 6 v. -7 r.

${ }^{21}$ Così sostiene PASCHETTO, 1984, p. 92.

${ }^{22}$ ARISTOTELIS, Metaphysica, II 3; Ethica Nichomachea, I 1; Analytica et posteriora,. I 30.

${ }^{23}$ PASCHETTO, 1984, p. 93.

${ }^{24}$ Cf. ARISTOTELIS, Metaphysica VI 2-3; Analytica et posteriora, II 12.

${ }^{25}$ Conc., f. 7 v.
} 
con l'occuparsi di medicina e i medici che praticano la loro arte in modo "più filosofico", che significa più scientifico, iniziano i loro studi dalla fisica ${ }^{26}$.

Infine all'obiezione secondo cui la medicina non si occupa di oggetti astratti, come dovrebbe fare un'autentica scienza, Pietro risponde esponendo la dottrina tradizionale dei tre gradi di astrazione, basata su una particolare interpretazione della classificazione delle scienze teoretiche contenuta in Metafisica VI 1. Secondo tale dottrina, la fisica astrae, cioè prescinde, solo dalla materia sensibile particolare (signata), non dalla materia sensibile comune, la matematica astrae dalla materia secondo la considerazione intellettuale, ma non secondo l'essere, e la metafisica astrae completamente dalla materia, sia quanto all'essere che quanto alla considerazione intellettuale. Il primo tipo di astrazione è praticato, secondo Pietro, anche dalla medicina, che dunque si occupa di oggetti astratti, come si addice ad un'autentica scienza ${ }^{27}$. È stato ormai riconosciuto che l'autore di tale dottrina non è Aristotele, ma Ammonio, il quale la introduce nel suo commento alla Isagoge di Porfirio usando il termine aristotelico "separato" (chôriston), reso in latino da Boezio come abstractum. Nella versione boeziana essa viene accolta da tutti i filosofi medievali e permane alla base dell'epistemologia scolastica sino ai giorni nostri (è ancora presente, ad esempio, in Jacques Maritain). L'inconveniente a cui essa va incontro è l'assimilazione, come oggetto della metafisica, dell'essere in quanto essere, considerato come del tutto astratto, cioè immateriale, alle sostanze immateriali (Dio, gli angeli e le anime razionali), assimilazione che darà luogo, con Duns Scoto, alla concezione dell'essere come concetto astrattissimo e quindi univoco, che sta alla base di gran parte della metafisica moderna (Suárez e la cosiddetta Schulmetaphysik, criticata da Kant) ${ }^{28}$.

In conclusione, nella sua trattazione della scientificità della medicina, esposta nel Conciliator, Pietro d'Abano si rifa anzitutto alla classificazione aristotelica degli abiti dianoetici, contenuta nell'Etica Nicomachea, e secondariamente alla classificazione aristotelica delle scienze, contenuta nella Metafisica, interpretando entrambe in modo fedele ad Aristotele, anche sulla base della dottrina della scienza contenuta negli Analitici posteriori: gli elementi non aristotelici che egli vi introduce sono la distinzione tra methodus compositiva e methodus resolutiva, derivata da Galeno e comune a tutta la cultura medievale, la dottrina dei tre gradi di astrazione, derivata da Ammonio e Boezio e anch'essa condivisa da tutta la cultura medievale, ed infine la concezione

\footnotetext{
${ }^{26}$ ARISTOTELIS, Parva naturalia (De sensu et sensibili) 436 a 17-b 1; (De respiratione) 480 b 23-30.

${ }^{27}$ Conc., f. 7 v.

${ }^{28}$ Per tutta questa materia si veda COURTINE, 1990.
} 
della medicina come arte e scienza, o come comprendente due parti, teorica e pratica. Solo quest'ultima è del tutto originale, cioè propria di Pietro, non tanto nella sua formulazione più generale, che probabilmente è rintracciabile anche in Aristotele, quanto nel modo preciso in cui egli la concepisce, a cui abbiamo accennato, ma su cui ritorneremo trattando dell'astronomia.

\section{LO STATUTO EPISTEMOLOGICO DELL'ASTRONOMIA NEL LUCIDATOR.}

Un discorso analogo a quello sulla medicina, fatto nel Conciliator, Pietro fa sull'astronomia nel Lucidator dubitabilium astronomiae, cioè nel libro "datore di luce" sui problemi dell'astronomia. La Differentia prima dell'opera è infatti interamente dedicata al problema "se l'astrologia con le sue appendici sia scienza" (i termini "astronomia" e "astrologia" nel medioevo era usati spesso in modo interscambiabile ${ }^{29}$, anche se, come vedremo, proprio Pietro d'Abano proporrà di tenerli distinti). Anche qui, come nel Conciliator, Pietro espone anzitutto gli argomenti contro lo statuto scientifico dell'astronomia, cioè in primo luoge il parere del Commentatore (Averroè), secondo cui l'astronomia odierna (huius temporis) non ha nessun valore per la realtà (in esse), ma serve solo ai calcoli (conveniens computazioni) ${ }^{30}$. Si tratta della nota polemica di Averroè contro l'astronomia tolemaica, divenuta dominante nel medioevo, la quale, avendo introdotto, per spiegare i moti apparentemente irregolari dei pianeti, epicicli ed eccentrici in luogo delle sfere omocentriche proposte nell'antichità da Eudosso ed accolte da Aristotele, sarebbe stata in contrasto con la struttura dell'universo sostenuta da Aristotele e considerata indiscutibile da Averroè. Per il Commentatore quindi l'astronomia tolemaica sarebbe servita solo a calcolare le posizioni dei pianeti, cioè avrebbe avuto soltanto il valore di un'ipotesi matematica (come in seguito l'astronomia copernicana per Osiander e Bellarmino). Pietro d'Abano dedicherà l'intero Lucidator a difendere l'astronomia tolemaica, con i suoi eccentrici ed epicicli, dalle obiezioni dei filosofi aristotelici ortodossi (Averroè e anche Tommaso d'Aquino, che la pensava come Averroè $)^{31}$, ma non è questo che ora ci interessa.

Altre obiezioni alla scientificità dell'astronomia si basano sulla dottrina aristotelica secondo cui la scienza è degli universali, mentre l'astronomia sarebbe di oggetti particolari (gli astri e i pianeti); sulla necessità di considerare

\footnotetext{
${ }^{29}$ Cfr. SELLER, p. 14-19.

${ }^{30}$ PIETRO D'ABANO, Lucidator, p. 106.

${ }^{31}$ BERTI, 2007.
} 
tutte le stelle, che sono migliaia, e non solo gli otto pianeti; sul fatto che gli oggetti dell'astronomia non sono cose buone e onorabili; sul fatto che spesso gli astronomi sono considerati dediti alla divinazione e derisi come incantatori; sul fatto che l'astronomia si occupa di generi di oggetti diversi (incorruttibili e corruttibili); infine sul fatto che essa non coincide con nessuna delle tre scienze teoretiche distinte da Aristotele nella Metafisica, cioè fisica, matematica e scienza divina. Contro queste obiezioni, quindi a favore della scientificità dell'astronomia, sta il fatto che essa era considerata una scienza da Aristotele e dai Caldei, e che si serve di dimostrazioni matematiche ${ }^{32}$.

Nel rispondere alle obiezioni Pietro anzitutto distingue l'astronomia, che si occupa dei moti degli astri, e l'astrologia, che invece formula giudizi (iudicia). Indi egli distingue tre modi di intendere la scienza, quello per cui essa procede dalle cause agli effetti, da lui identificato con la doctrina compositiva, quello per cui essa procede dagli effetti alle cause, da lui identificato con la doctrina resolutiva, e quello improprio e comune, per cui essa è semplicemente comprensione della verità. Siamo di fronte, come si vede, esattamente alle stesse considerazioni fatte nel Conciliator a difesa della scientificità della medicina. In seguito Pietro richiama la classificazione delle scienze esposta da Aristotele nel VI libro della Metafisica, affermando che ogni scienza è theorica, activa o factiva. Le scienze del primo tipo si occupano di oggetti che sono opera della natura, mentre le altre si occupano di opere dell'uomo, cioè di azioni o di produzioni. Queste ultime sono identificate da Pietro con le artes, quali l'agricoltura, la medicina, la fabbricazione della lana, ecc., e l'arte è definita con Aristotele recta ratio factibilium ${ }^{33}$. Interessante è poi l'identificazione delle scienze pratiche (activae) con la prudentia, abito della ragione pratica illustrato nel VI libro dell'Etica Nicomachea, la quale è a sua volta divisa in ethica monastica, yconomica seu domus dispensativa e politica vel civilis ${ }^{34}$, identificazione che non tiene conto della diversità affermata da Aristotele tra scienza e prudenza, mentre la triplice divisione risale ai commentatori neoplatonici di Aristotele ed è consueta nella cultura medievale.

Pietro riprende poi la classificazione aristotelica delle scienze teoretiche, basandola, come ormai era tradizione, sulla dottrina dei tre gradi di astrazione, per cui la fisica tratta di oggetti separati solo dalla materia individuale, non da quella sensibile comune (primo grado di astrazione), le matematiche trattano di

\footnotetext{
${ }^{32}$ Luc., p. 107-108.

${ }^{33}$ Luc., p. 109-111.

${ }^{34}$ Luc., p. 112.
} 
oggetti separati dalla materia sensibile, non da quella intelligibile (secondo grado di astrazione), e la metafisica, o filosofia prima, o scienza divina, tratta di oggetti separati da qualunque materia (terzo grado di astrazione). La debolezza di questa dottrina, peraltro non evidenziata da Pietro, è il fatto che, in base ad essa, egli stesso attribuisce alla scienza divina la trattazione della sostanza e degli accidenti, cioè di oggetti che non hanno nulla di divino, ma che costituiscono la divisione fondamentale dell'essere in quanto essere ${ }^{35}$. In tal modo l'essere in quanto essere viene posto sullo stesso piano delle sostanze immateriali, come se entrambi fossero oggetti della metafisica allo stesso titolo, mentre l'essere in quanto essere è oggetto nel senso che è ciò che deve essere spiegato (explanandum), e le sostanze immateriali sono oggetto nel senso che sono ciò che spiega (explanans).

A proposito delle matematiche, Pietro fa notare come sia l'aritmetica che la geometria comprendano una parte teorica, riguardante rispettivamente i numeri e le figure, e una parte pratica, riguardante rispettivamente i calcoli e le misurazioni. Della parte pratica di entrambe le scienze si servono i cultori delle scienze ad esse subalterne, cioè armonica, ottica e astronomia. Quest'ultima, come già detto, si occupa dei moti degli astri, e si distingue dall'astrologia, che invece formula giudizi e perciò è detta iudicativa o iudicialis.

Per quanto riguarda la posizione di scienza subalterna alle matematiche, assegnata all'astronomia, Pietro non fa che riprendere Aristotele. Del tutto originale è invece il suo discorso concernente l'astrologia, cioè la scienza giudicativa o giudiziale. Quest'ultima, a suo dire, risale a Tolomeo ed è stata ripresa dagli astrologi arabi, quali Albategni, Alfargano, Arzachel, Alkindi e molti altri. Essa si divide a sua volta in due parti, una introductiva in iudicia, che insegna al giudicante a precognoscere necessaria, cioè a prevedere ciò che accade di necessità, e l'altra exercitativa, grazie alla quale possiamo giudicare e scendere nei casi particolari, cioè finalmente per canones sanativos actualiter curare, ossia curare le malattie. L'astrologia giudiziale in tal modo si estende sino a comprendere l'arte di curare, cioè la medicina. La parte exercitativa di essa sembra essere per Pietro la più interessante, perché egli la divide a sua volta in quattro parti, la prima concernente le "rivoluzioni" dei pianeti, cioè le loro congiunzioni o opposizioni nei diversi momenti dell'anno; la seconda concernente le "nativită", cioè gli eventi che incombono sui nati in un certo momento dell'anno; la terza concernente le "interrogazioni", cioè le domande volte a sapere in quale momento conviene prendere una certa decisione; la quarta concernente le "elezioni", cioè le decisioni lodevoli in base a una certa

${ }^{35}$ Luc., p. 113. 
disposizione degli astri. A quest'ultima è sottoposta la scienza delle "immagini astronomiche", che raffigurano i caratteri, le attitudini, le mansioni dei nati nei diversi momenti dell'anno ${ }^{36}$.

Sul significato di questa dottrina, che costituisce la parte più originale del pensiero di Pietro d'Abano, esiste una letteratura ormai cospicua, che ha messo in luce come egli intenda l'astronomia in un senso che oggi considereremmo assolutamente scientifico, perché basata su cause del tutto naturali, e tuttavia le attribuisca importanti compiti pratici, come quello di prevedere il futuro, di consigliare sulle decisioni da prendere, di curare le malattie. Gli studi pionieristici in questa direzione sono quelli di Graziella Federici Vescovini, contenuti anzitutto nelle introduzioni alle varie Differentiae del Lucidator, ma poi anche in altri saggi ${ }^{37}$, a cui ne sono seguiti altri di ottimo livello, come quello di Fabio Seller ${ }^{38}$.

Nel seguito della Differentia prima Pietro spiega che gli astri, formati di materia incorruttibile, svolgono il ruolo di cause intermedie tra la causa prima di tutto, che è Dio, e gli eventi che accadono sulla terra. Egli biasima i teologi (quidam divini) che ipocritamente vogliono far dipendere ogni cosa immediatamente da una decisione divina, trascurando il fatto che Dio agisce per mezzo delle sue creature, le quali in tal modo manifestano la potenza del Creatore, come dice l'apostolo Paolo nella Lettera ai Romani. Perciò li chiama irrationales, pusillanimes et fortuitos, perché non comprendono che l'astrologo, spiegando il significato degli astri, può evitare molti mali ${ }^{39}$. Questi accenti forse spiegano il motivo dei vari processi che Pietro dovette subire, senza che ci sia bisogno di ammettere da parte sua alcuna attività di mago o di negromante ${ }^{40}$. Egli infatti spiega che le stelle esercitano la loro causalità anche sugli individui, ma non in modo deterministico, perché - come dice Aristotele nel VI libro della Metafisica - esiste anche l'accidente, ovvero il caso, la fortuna, che costituisce la parte eccedente, e quindi estranea, rispetto all'agire "per lo più" delle cause naturali. La scienza quindi, nella fattispecie l'astrologia, può prevedere ciò che accade necessariamente o per lo più, non ciò che accade raramente ${ }^{41}$.

\footnotetext{
${ }^{36}$ Luc., p. 114-117.

${ }^{37}$ FEDERICI VESCOVINI, 2013.

${ }^{38}$ SELLER, 2009, p. 9-83.

${ }^{39}$ Luc., p. 123-125.

${ }^{40}$ Sui diversi giudizi cui andò soggetto Pietro d'Abano presso i posteri, si veda PIAIA, 1983.

${ }^{41}$ Luc., p. 126-127.
} 
Sempre nell'intento di mostrare che l'astronomia è una scienza, nel senso aristotelico del termine, Pietro afferma che il suo oggetto, cioè gli astri, forma un ens per se, del quale possono essere dimostrate le proprietà e le passioni. Tra queste c'è, come proprietà di ogni corpo celeste, la passione detta da Pietro applicare, in virtù della quale ciascun astro, in quanto è animato ed eternamente mosso, è applicabile, ossia - afferma Pietro - causativum ac ostensivum ${ }^{42}$. Seller spiega che il termine applicare connota la funzione per cui gli astri agiscono in qualità di cause sugli enti sublunari, e questi accolgono l'influsso astrale in quanto id cui applicatur ${ }^{43}$. Pietro infatti - afferma Seller - si riallaccia alla tradizione che vede gli astri come cause che esercitano il loro influxus sugli enti sublunari, ma anche a quella secondo cui gli astri potevano significare sui fatti del mondo. Graziella Federici Vescovini, a sua volta, spiega che questa proprietà allude all'attività del corpo incorruttibile e mobile, cioè il cielo, la quale "si applica" ai corpi inferiori e corruttibili, tra i quali il corpo umano, che risulta pertanto applicatum. In quanto soggetto a questa azione causale da parte degli astri, il corpo umano risulta a sua volta sanabile, cioè curabile $^{44}$. Si ottiene così una perfetta compenetrazione e continuità tra astrologia e medicina, le due scienze che più di tutte interessano Pietro.

La parte dell'astronomia che ha a che fare con i corpi incorruttibili, cioè con gli astri e con i loro movimenti, ossia la parte teorica, detta propriamente "astronomia", è secondo Pietro una scienza certa, non congetturale, in quanto si serve delle dimostrazioni matematiche, cioè è una scienza subalterna alla matematica. Invece la parte che concerne i corpi corruttibili, cioè la parte pratica, o "giudiziale", detta propriamente "astrologia", avendo a che fare con oggetti mutevoli, è subalterna non alla matematica, ma alla fisica, e quindi è meno certa della prima, e più difficile da acquisire perfettamente. Ma non per questo bisogna pigramente posporla, perché anzi si deve cercare di sapere quanto è possibile di essa, a causa della sua grande utilità ${ }^{45}$. Essa è una scienza "nobile", per la nobiltà del suo oggetto, difficile e laboriosa, cioè faticosa, ma tuttavia utile, anzi necessaria, superiore alle altre scienze, e soprattutto licita, cioè "lecita", perché non contiene nulla che contrasti con le leggi divine. Detto questo, Pietro si lancia in una nuova invettiva contro coloro che, fingendosi ipocritamente devoti (deicolos), detestano l'astronomia, soprattutto quella giudiziale, in quanto

\footnotetext{
42 Luc., p. 133-134.

43 SELLER, 2009, p. 57, che cita anche Luc., p. 151.

${ }^{44}$ FEDERICI VESCOVINI, 2013, p. 13.

${ }^{45}$ Luc., p. $135-140$.
} 
ritengono che essa ammetta un determinismo universale e che deroghi alla onniscienza divina, pretendendo di preconoscere il futuro ad essa riservato ${ }^{46}$.

Infine Pietro riconosce che le accuse di non scientificità rivolte all'astronomia riguardano soprattutto la parte giudiziale di essa, "la quale al tempo di Aristotele non era ancora perfetta, come infine divenne grazie a Tolomeo e agli arabi, i quali si impegnarono diligentemente intorno ad essa" ${ }^{\prime 7}$. Nel complesso quindi si può dire che, anche mediante la collocazione dell'astronomia tra le matematiche applicate, Pietro d'Abano segue Aristotele, come lo segue per l'intera classificazione delle scienze, aggiungendovi, come tutti facevano nel medioevo, la dottrina dei tre gradi di astrazione e la distinzione tra metodo compositivo e metodo risolutivo. In particolare egli segue Aristotele nell'attribuire all'astronomia il carattere di scienza di ciò che accade "per lo più", attribuito dallo Stagirita alla fisica, tra le scienze teoretiche, e alla "scienza politica”, tra quelle pratiche. Del tutto originale è invece la distinzione, all'interno dell'astronomia, come della medicina, di una parte teorica e una parte pratica, che Pietro stesso fa derivare non da Aristotele, ma da Tolomeo e dagli arabi ${ }^{48}$.

BERTI, Enrico. The Aristotelian classification of the sciences in Peter of Abano. Trans/Form/ Ação, Marília, v. 37, n. 3, p. 17-32, Set./Dez., 2014.

\begin{abstract}
Peter of Abano's classification of the sciences is an interesting reformulation of the analogous classification proposed by Aristotle in Metaphysics VI, and of Aristotle's theory of the dianoetic habits found in Nichomachean Ethics VI. As apparent in the Conciliator for medicine and in the Lucidator for astronomy, Peter follows the Aristotelian classification and the interpretations of it given in Middle Ages (doctrine of the three degrees of abstraction, distinction between methodus compositiva and methodus resolutiva), adding as his own original contribution the introduction of a practical part on medicine and on astronomy (astronomia iudicialis), the latter derived from Ptolemy and the Arabs.
\end{abstract}

KEYWORDS: Aristotle. Science. Art. Medicine. Astronomy. Astrology.

\title{
BibLIOGRAFIA
}

ARISTOTELIS. Ethica Nicomachea. Rec. I. Bywater. Oxford: Clarendon Press, 1894.

${ }^{46}$ Luc., p. 141-148.

${ }^{47}$ Luc., p. 151: "respectu partis iudicialisnque, tempore Aristotelis, nondum erat perfecta, ut demum a Ptolomeo et arabicis circa illam diligenter laborantibus".

${ }^{48}$ Sull'epistemologia di Pietro d'Abano si veda anche BOTTIN, 2013. 
Parva naturalia. Rec. W.D. Ross. Oxford: Clarendon Press, 1955.

. Metaphysica. Rec. W. Jaeger. Oxford: Clarendon Press, 1957.

. Analytica priora et posteriora. Rec. W.D. Ross. Oxford: Clarendon Press, 1964.

fragmenta.

. Opera. Ed. O. Gigon. Berlin: W. de Gruyter, 1987. v. 3: Librorium deperditorum

BERTI, Enrico. Pietro d'Abano, Giotto e Dondi dell'Orologio. In: LONGO, O. (Ed.). Padua felix. Storie padovane illustri, a cura di O. Longo. Padova: Esedra, 2007. p. 75-84.

. Differenza tra il metodo risolutivo degli aristotelici e la resolutio dei matematici. In: . Nuovi studi aristotelici. Brescia: Morcelliana, 2009. v.4/1: L'influenza di Aristotele.

Antichità, Medioevo e Rinascimento. p. 255-271.

BOTTIN, Francesco. Peter of Abano: the scientific method of a "diligens indagator". Medioevo, v. 37, p. 319-359, 2013.

COURTINE, Jean-François. Suárez et le système de la métaphysique. Paris: Presses Universitaires de France, 1990.

FEDERICI VESCOVINI, Graziella. La médecine, synthèse d'art et de science selon Pietro d'Abano. In: RASHED, R.; BIARD, J. (Eds.). Les doctrine de la science de l'antiquité à l'âge classique. Leuven: Peeters, 1999. p. 237-256.

. L'astrologie comme science théorique, rationnelle et autorisée dans le Lucidator de Pietro d'Abano. In: BOUDET, J.-P.; COLLARD, F.; WEILL-PAROT, N. (Eds.). Médecine, astrologie et magie entre Moyen Âge et Renaissance: autour de Pietro d'Abano. Firenze: Edizioni del Galluzzo, 2013. p. 3-20.

PASCHETTO, Eugenia. Pietro d'Abano medico e filosofo. Firenze: Vallecchi, 1984.

PETRI DE ABANO PATAVINI. Conciliator differentiarum philosophorum, \& praecipue medicorum. Venetiis: apud Iuntas, 1565. Ristampa a cura di E. Riondato e L. Olivieri, Padova, Editrice Antenore, 1985.

PIAIA, Gregorio. L'immagine di Pietro d'Abano nella storiografia filosofica e scientifica del Sei-Settecento. In: . Vestigia philosophorum. Rimini: Maggioli, 1983. p. 199-232.

PIETRO D’ABANO. Lucidator dubitabilium astronomiae. Ed. Graziella Federici Vescovini. Padova: Programma e 1+1 Editori, 1988.

RENAN, Ernest. Averroès et l'averrö̈sme. Paris: M. Levy Frères, 1861.

SELLER, Fabio. Scientia astrorum: la fondazione epistemologica dell'astrologia in Pietro d'Abano. Napoli: Giannini Editore, 2009.

Enviado em: 05/09/2014

Aceito em: 26/09/2014 